\title{
Absolute Light-Scattering Photometer: I. Design and Operation
}

\author{
Donald McIntyre and G. C. Doderer ${ }^{1}$
}

\begin{abstract}
A new light-scattering photometer has been designed and built for determining the absolute scattering from polymer solutions. The instrument is also capable of performing as a research instrument for making measurements at very low and very high angles, and at very low and very high intensities of scattered light. The instrument scans the angular scattering either manually or automatically while measuring continuously the ratio of the scattered light to the incident light.
\end{abstract}

\section{Introduction}

The general need for accurate light-scattering measurements for the determination of molecular weights of high polymeric compounds is well known. ${ }^{2}$ Some of the problems connected with these measurements and most of the work up until 2 years ago have been summarized in two publications. ${ }^{34}$ The present work was done with the object of increasing the accuracy of determining molecular weights of macromolecules rather than that of determining optical constants of materials.

The instrument described here was designed to attain an accuracy of 3 to 5 percent in the determination of molecular weights. An instrument that must be versatile enough to make molecular weight measurements in any research problem and also make absolute scattering determinations imposes many compromises in design. For example, a closed-in system is much more convenient for general laboratory work, whereas an open system in a darkroom would be more suitable for absolute measurements. A compromise is to make a rather large working compartment in the instrument. Also, an absolute measurement would only have to be carried out at a few fixed angles so that considerably more rigidity and inflexibility could be built into an instrument to attain more accurate measurements. In contrast, a research instrument must be capable of scanning a large angular range with a measuring technique that is not unduly time consuming and fatiguing.

With these thoughts in mind an instrument was constructed with emphasis on providing (1) a variety of precise experimental setups involving different combinations of sources, lightbeams, filters, sample cells, and receiver arrangements; (2) a well-collimated beam of monochromatic light having uniform intensity and producing negligible stray light inside the instrument; (3) a sensitive and stable electronic

\footnotetext{
1 Guest worker at the National Bureau of Standards from the Office of the Surgeon General, Department of the Army. Present address: General Electric Co., Louisville, Ky.

2 H. P. Frank and H. Mark, J. Polymer Sci. 17, 1 (1955).

3 K. A. Stacey, Light Scattering in Physical Chemistry (Academic Press, New York, N.Y., 1956)

4 M. M. Fishman, Light Scattering by Colloidal Systems, An Annotated Bibliography, Technical Service Laboratories, River Edge, N.J., 1957 (also additional annual supplements).
}

detecting and recording system; and (4) a rugged mechanical design featuring easily adjusted components.

\section{Apparatus}

\subsection{General Description}

The over-all plan of the instrument is shown in figure 1. A transformer connected to a voltage regulator operates a mercury vapor lamp to produce a small intense source of light. The image of the source is then projected as a monochromatic beam of variable collimation that has well-defined dimensions at the center of the cell $(\mathrm{C})$. From there the beam passes on to a light trap where it is absorbed.

A very small percentage of the projected beam is reflected by a cemented glass prism on to a monitoring photomultiplier tube (Mon PM). The measuring photomultiplier tube (Meas PM) is mounted on a T-beam connected to a goniometer so that it can be rotated to view the light scattered from the sample, which is mounted on a stationary pedestal at the center of rotation. Both phototubes are similar and operate from the same power supply controlled by a voltage regulator. The phototube outputs are amplified and fed to a ratio recorder

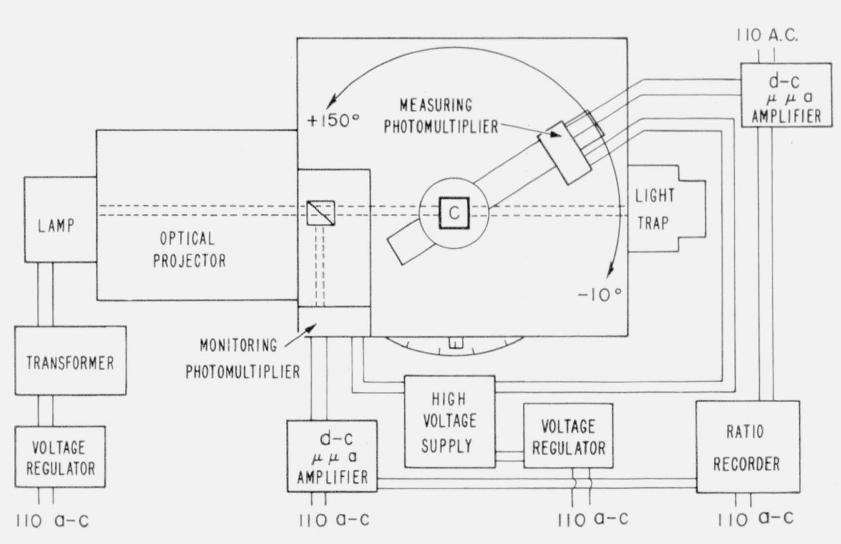

Figure 1. Schematic diagram of photometer. 
which is essentially an automatic precision potentiometer operating a chart recorder. The recorder compares the voltage from the measuring circuit with the reference voltage from the monitoring circuit. Thus the amount of scattered light is measured relative to the incident beam and is recorded as a value which is independent of the absolute beam intensity.

Figure 2, an exterior view of the instrument, shows the source enclosure, optical projector, and goniometer housing mounted on a table and connected to the electronic control panel.

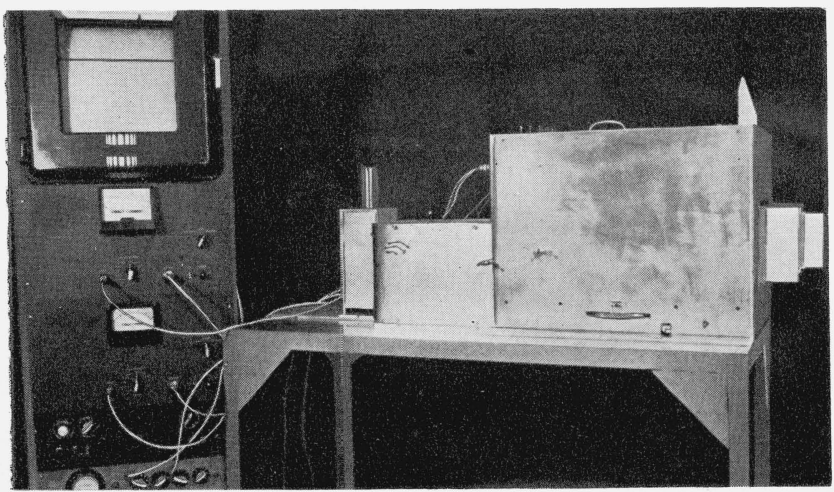

Figure 2. Overall view of phutometer.

\subsection{Optical System}

The essential optical components are represented in figure 3. Two source lamps have been tested: an $85-\mathrm{w}$ mercury vapor lamp, and a $250-\mathrm{w}$ compactsource medium-pressure mercury vapor discharge lamp. The latter forms a discharge approximately $3.75 \mathrm{~mm}$ high by $1.5 \mathrm{~mm}$ wide between two chiseledge electrodes. The chisel edges are alined in the direction of the optic axis so that the arc wanderings tend to occur along, rather than across the axis.

The source is located at the focal point of a planoconvex condensing lens $L_{1}$ of $84.5 \mathrm{~mm}$ focal length which receives light through a wide stop $\mathrm{S}_{1}$ and a
Corning 4600 special heat absorbing filter $\mathrm{F}_{1}$. Essentially parallel light from lens $\mathrm{L}_{1}$ passes through removable filters $\mathrm{F}_{2}$ and $\mathrm{F}_{3} . \mathrm{F}_{2}$ represents three monochromatic filter combinations: (1) Corning glass filters 3389 and 5113 passing mercury blue light of wavelength $436 \mathrm{~m} \mu$; (2) Corning glass filters 3484, 5120 , and 4303 passing mercury green light of wavelength $546 \mathrm{~m} \mu$; and (3) a multilayer interference filter also passing the mercury green line. $\mathrm{F}_{3}$ represents $1 \mathrm{~mm}$ thick optical neutral glass filters, as do $\mathrm{F}_{4}$ and $\mathrm{F}_{5}$.

A second condensing lens $\mathrm{L}_{2}$, similar to $\mathrm{L}_{1}$, focuses an image of the source on aperture $A_{1}$ located at the focal point of lines $\mathrm{L}_{3}$. The aperture $A_{1}$ is fitted into a milled slot and is removable so that variable degrees of beam collimation are obtainable. The largest aperture is $3.4 \mathrm{~mm}$ high by $1 \mathrm{~mm}$ wide so that it is smaller than the source image. Field stops which determine the size and shape of the projected beam may be inserted at position $\mathrm{S}_{2}$ and adjusted axially so that the achromatic collimating lens $\mathrm{L}_{3}$ of $98.6 \mathrm{~mm}$ focal length and 40-mm diameter focuses a sharp image of the field stop in the center of the sample cell, C. The beam can be shut off at the entrance to the main instrument housing by shutter $\mathrm{Sh}_{1}$. The relative positions of the above components may be seen in figure 4 . The remaining optical components inside the main housing are shown also in figure 5 .

A 1-in. glass square consisting of two $45^{\circ}-90^{\circ}$ prisms cemented together with Canada balsam is indicated as $\mathrm{G}$ in figure 3. The cemented interface reflects a very small percentage of the incident light into the monitoring system. By choosing glass and cement of nearly the same refractive index, the same beam as that viewed by the measuring phototube may be monitored with minimum intensity loss and negligible polarization. Using barium crown glass of $n_{d}=1.5415$, which was obtained from samples made at the NBS glass plant, and Canada balsam, which was reported to have an $n_{d}=1.5446$, the measured reflectance from the surface was $10^{-5}$ of the incident green light. Thus the match of the refractive indices at this wavelength is closer than the above figures would indicate.
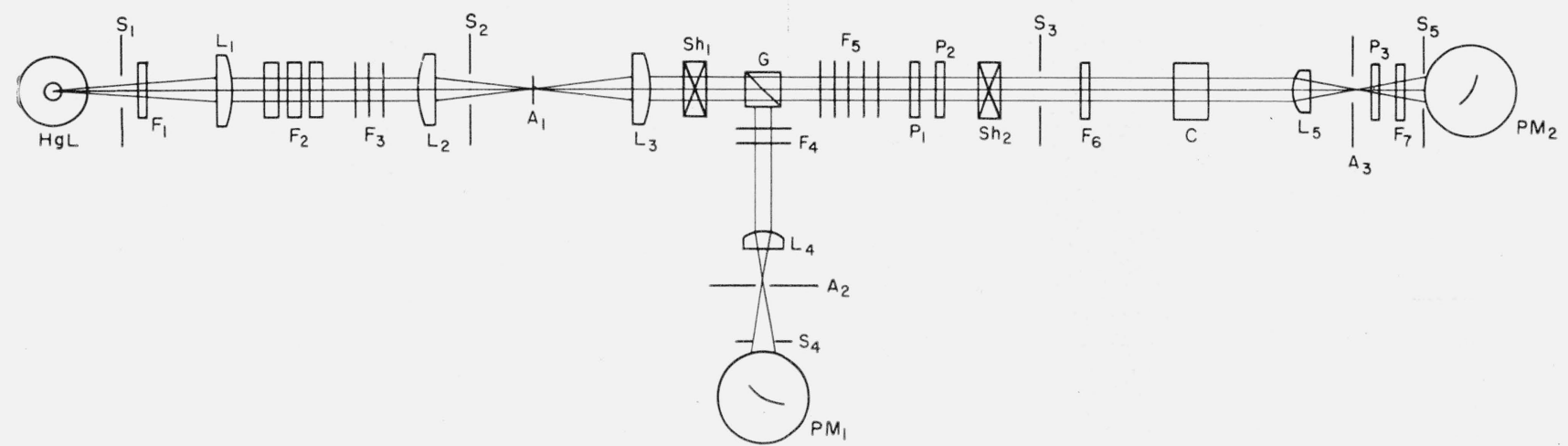

Figure 3. Schematic diagram of the optical system. 


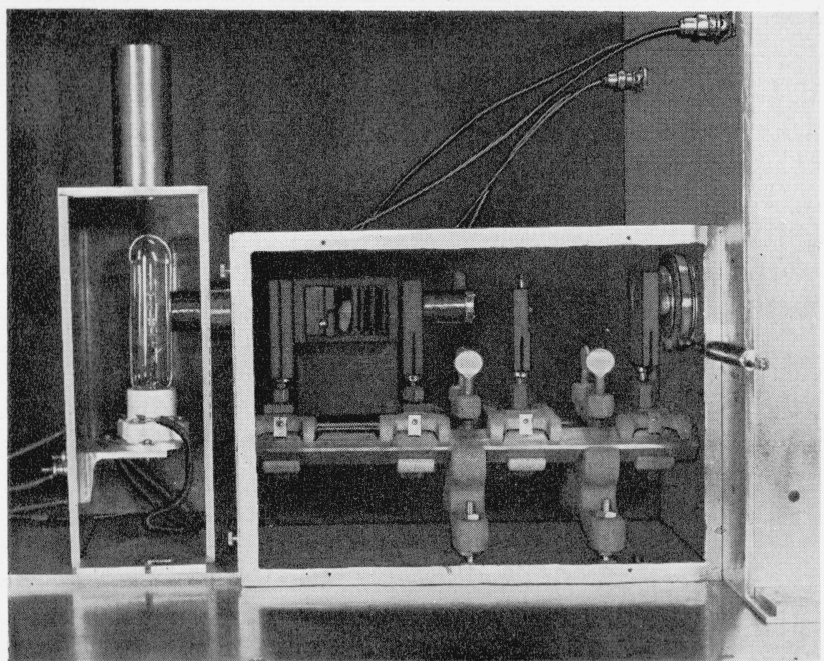

Figure 4. Detailed view of the projector.

The main beam is attenuated by movable filters $\mathrm{F}_{5}$ having transmittances of approximately $1 / 2,1 / 3$, $1 / 10,1 / 40$, and $1 / 4,500 . \mathrm{P}_{1}$ and $\mathrm{P}_{2}$ are Polaroid KN36 glass-laminated filters mounted with axes horizontal and vertical, respectively, and having maximum deviation of $4 \mathrm{~min}$ and maximum resolving power of $1 \mathrm{~min}$. Shutter $\mathrm{Sh}_{2}$ allows the measuring beam to be shut off without disrupting the monitoring system. $\mathrm{S}_{3}$ is a baffle to eliminate stray light. In addition, removable baffles can be inserted into this nosepiece arrangement as beam dimensions are changed. Another tube can also be inserted on the cell table which is capable of accommodating two additional baffles. Thus there are four possible interchangeable baffles in addition to two shutter diaphragms, which are circular for eliminating stray light. A 4-mm-thick neutral glass filter $\mathrm{F}_{6}$ of approximately $1 / 30,000$ transmittance can be mounted on the end of the measuring phototube arm and subsequently brought into the lightbeam when the phototube is rotated into the beam. This is mounted as a milled slot so that other neutral filters can be inserted as they are needed. This arrangement is used only for automatic scanning of the entire angular scattering, and for special purposes of calibration.

The receiver optics consist of an achromatic lens $\mathrm{L}_{5}$ of $40.5-\mathrm{mm}$ focal length and $18.5-\mathrm{mm}$ diameter, a small aperture $A_{3}$ mounted at the focal plane, and a stop $\mathrm{S}_{5}$ which determines the area of lightbeam that is to be admitted to the phototube $\mathrm{PM}_{2}$. There is a series of aperture stops, $\mathrm{A}_{3}$, that can be easily and quickly inserted to define the angular acceptance of the receiver, just as there is a series of field stops, $\mathrm{S}_{5}$, that can be inserted into the receiver in order to change the field of view consonant with the physical limitations of the phototube. The position of the whole receiver is adjustable so that the image of the field stop can be placed directly at the center of rotation. A Polaroid HN22 filter

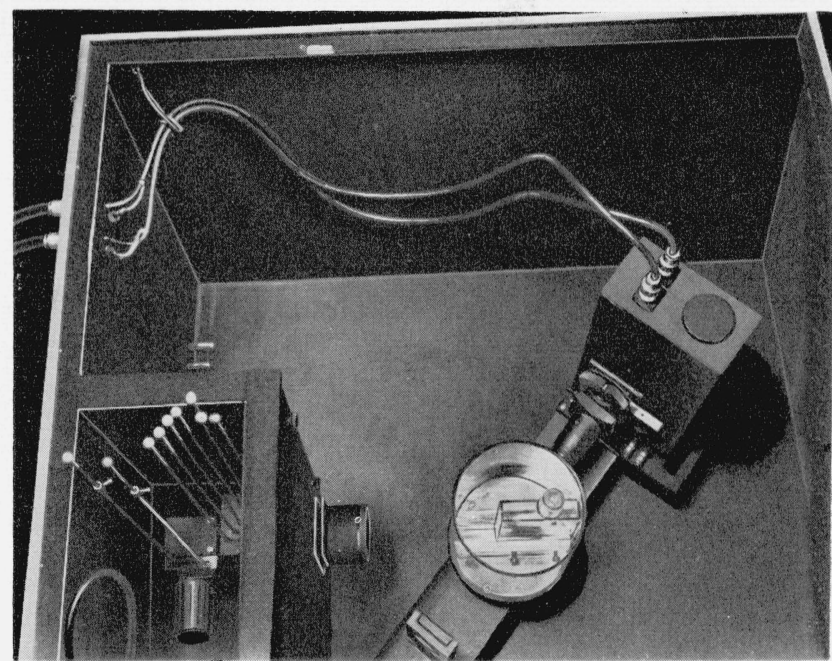

Figure 5. Detailed view of receivers and cell table.

$\mathrm{P}_{3}$ is mounted in a slide and can be adjusted to rotate $90^{\circ}$ between the axes of the polarizers. A second slide $\mathrm{F}_{7}$ may be used to hold a fluorescence filter. In addition, provision is made to insert a diffusor behind the field stop when necessary.

The monitoring optics consist of lens $\mathrm{L}_{4}$, aperture $\mathrm{A}_{2}$, field stop $\mathrm{S}_{4}$, and phototube $\mathrm{PM}_{1}$, similar to those in the measuring receiver just described. Attenuating filters $\mathrm{F}_{4}$ having transmittances of approximately $1 / 6$ and $1 / 36$ are also placed before this receiver.

There are two interchangeable phototube housings on the measuring photo tube. Each of these housings can accommodate the receiver mount described, and can be placed into position without significant changes by means of milled slots. One housing contains a 1P21 photomultiplier which is used routinely, while the other housing contains an end-on photomultiplier, 5819, for special work. In addition, the latter housing can take a cassette which uses 1 by 3 in. photographic plates in place of the photomultiplier.

\subsection{Electronic System}

The sensitive and stable detecting-recording system is based upon the use of matched precision components in the monitoring and measuring circuits which operate the ratio recorder. Optionally, the recorder may be operated as a typical potentiometer recorder to indicate the output of either phototube separately. The receivers are closed desiccated metal housings containing RCA 1 P21 multiplier phototubes. A power supply having 1-ma d-c output, stabilized to 0.5 percent and fed by a $0.250-\mathrm{Kva}$ voltage stabilizer, is used for the high-voltage supply. When operated at $800 \mathrm{v}$ d-c the phototube dark currents are about $3 \times 10^{-10}$ amps. The monitor light beam may be adjusted to its maximum unfiltered intensity, which produces a total output of over $1 \times 10^{-6}$ amps. Thus dark-current fluctuations have a negligible effect on the final monitor reference voltage. In 
practice, a monitor output of about $1 \times 10^{-8}$ amp has proven very satisfactory. The phototube outputs are amplified by two Leeds and Northrup 9836A stabilized d-c micromicroampere indicating amplifiers providing a full scale output of $10 \mathrm{mv}$ over a range of $1 \times 10^{-9}$ to $2 \times 10^{-6}$ amp with an accuracy of \pm 0.5 percent of the range or $0.5 \times 10^{-11}$ amp at the most sensitive region. The measuring circuit amplifier supplies voltage to the potentiometer of a Speedomax ratio recorder where it is compared with the monitor reference voltage to an accuracy of 0.3 percent of the range.

Thus fluctuations in source intensity affect both input voltages proportionately, and the final recorded value is independent of the absolute beam intensity. In operation, the measured values are corrected by subtracting a dark current reading with the shutter $\mathrm{Sh}_{2}$ closed. This is done either arithmetically or by the use of a biasing voltage on the measuring phototube signal using a mercury cell.

The 85-w lamp operates from a transformer on $110 \mathrm{v}$ a-c; whereas, the 250-w lamp operates from a ballast on a $220 \mathrm{v}$ a-c line.

\subsection{Mechanical Elements}

The instrument was constructed as three independently adjustable assemblies; namely, the source, the optical projector, and a light-tight measuring compartment mounted on a heavy welded steel framework table top. All of these pieces were optically alined using the center of rotation of the measuring phototube and goniometer as a fiducial mark. Visual observations of the beam by means of targets placed in indexed positions along the rotating beam and center of rotation were used for the final alinement. Although the overall construction is sturdy to minimize possible vibration or movement, a wide range of adjustability is maintained. Thus the receiver apertures and field stops thread into place against positioning rings, and the mounts are positioned with the aid of pins.

The measuring phototube housing is mounted in machined ways on an aluminum beam of large $T$ cross section. With a narrow slit in front of the receiver lens, the entire housing may be moved across the lightbeam by a screw to determine the uniformity of the light flux. The aluminum beam is connected to a goniometer which is graduated in degrees from $-10^{\circ}$ to $+150^{\circ}$ with respect to the incident light. The assembly may be rotated manually as desired, or automatically in either direction by a reversible geared motor drive. A scanning rate of $40^{\circ} / \mathrm{min}$ was used to synchronize with the chart recorder speed of 2 in./min. Two electrical limit switches prevent mechanical damage due to overscanning and a third microswitch is operated by pins spaced $10^{\circ}$ apart on the goniometer to activate a magnetic pen marker which produces a fiducial mark on the chart.

Sample cells and thermostats are positioned accurately on a stationary pedestal at the center of rotation by a three-point support system. The pedestal top has three $1 / 4$-in.-diam ball bearings on $3 / 16$-in.-diam holes spaced $120^{\circ}$ apart on a 3 -in.-diam circle centered on the axis of rotation. Each cell assembly is cemented to a similarly drilled plate which automatically indexes on the pedestal with no undesirable mechanical tight fits or fastenings.

The light trap has two sections containing dark neutral filter glasses mounted at $45^{\circ}$ to the incident beam. These filters at this angle of incidence absorb most of the light, reflecting only a few percent from their surfaces. In the large first section which absorbs low intensity scattered light surrounding the main beam, the filter surfaces reflect any light onto the black-painted trap walls where further reflection is negligible. The center of the first section is open and passes the main beam into the second section.

In this section the light undergoes two absorptions and $45^{\circ}$ reflections by filter glass until it is finally directed to a black-painted portion of the trap not visible to the interior of the instrument compartment. The entire light trap is removable so that the interior may be viewed by a camera or telescope.

The photometer is also capable of operating at high temperatures since the cell table fixtures are located only by means of ball bearings which partially insulate the shaft below from the cell. Also the individual photometer housings are water cooled on the front plate to prevent any increase in the dark current by radiation from the sample.

\section{Performance}

\subsection{Electronic}

The 250-w lamp provides a light intensity approximately 11 times that of the 85-w lamp, but the arc is relatively unstable and would produce unacceptable errors in a single channel instrument, as is shown in the upper portion of the recorder trace in figure 6 . The lower portion shows a trace under identical conditions, but using the monitored ratio-recording system. Here the trace variations are reduced to the small noise inherent in a high level d-c amplification system. From such a trace a constant average value is easily read.

In order to determine the response characteristics of the measuring system over a large range of intensities the transmittance of a neutral filter of approximately $1 / 2$ transmittance was determined while reducing the lightbeam intensity with a neutral filter. In this work a large viewing field was used on the photomultiplier to eliminate the small inevitable local variations in a neutral filter. In changing the intensity by a factor of 1,000 , the measured transmittance of the filter showed a maximum deviation of less than 1 percent with an average deviation of less than 0.5 percent. This is a severe test because in the very low intensity measurements the signal is much less than that from pure liquids and the ratio of dark current to total current becomes much larger.

Over several hours the system is very stable; however, the ratio of scattered light from an object will eventually change due to a small change of the temperature of the cemented prism. The small change 


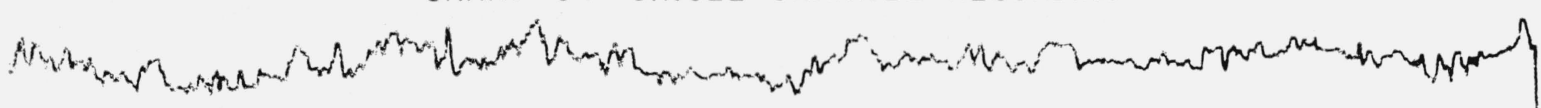

CHART 35 RATIO RECORDING

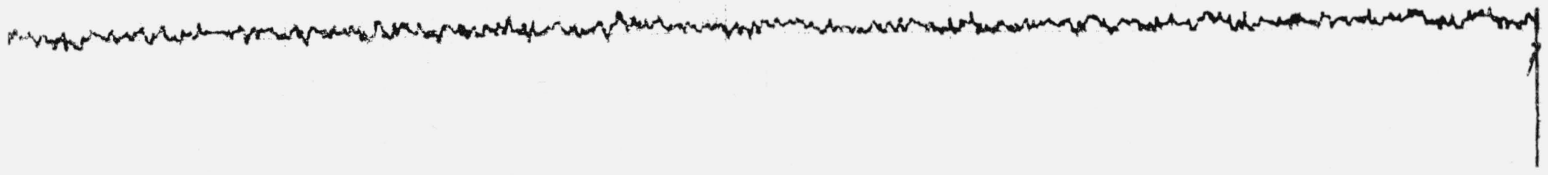

F1GURE 6. Photometer irace of scattered light from a diffuser with (1) a single measuring photomultiplier, and ( 2$)$ a ratio of two photomultiplier signals.

in temperature in the prism can affect the percentage of reflected light so that the ratio can change. This is not critical since all measurements are made in the course of a few hours, and those kinetic measurements which last longer can easily be referred to the direct beam whether the scanning is manual or automatic.

\subsection{Photometric}

Stray light. In the early experiments observations were made to determine the existence of stray light which might be caused by some unforeseen peculiarity of the apparatus. Thus experiments were conducted with a nosepiece containing two stops which allowed the phototube to view a 6 - by $10-\mathrm{mm}$ field while accepting about $2.5^{\circ}$. The projected beam was about $10 \mathrm{~mm}$ wide and $12 \mathrm{~mm}$ high with a divergence of $0.5^{\circ}$ in the horizontal direction and $1^{\circ}$ in the vertical direction. Measurements were initially made shortly after changing stops with the box open. The photomultiplier current is measured as a function of viewing angle and of time after closing the top of the instrument. The data are listed in table 1 . Below $12^{\circ}$ the phototube receiver by visual observation appears to enter the edge of the large beam. The current in excess of the dark current can be attributed to stray light. From these results it appears that unless measures are taken to allow all of the dust to settle in the box the results in the forward direction will always contain a large amount of scattered light.

TABLE 1. Photomultiplier currents at different angles and times

\begin{tabular}{|c|c|c|c|}
\hline \multirow{2}{*}{ Angle } & \multicolumn{3}{|c|}{ Current in amperes } \\
\hline & $1 \mathrm{hr}$ & $1 \mathrm{day}$ & 2 days \\
\hline $\begin{array}{l}150 \\
120 \\
90 \\
60\end{array}$ & $\begin{array}{l}22.7 \times 10^{-11} \\
17.6 \\
18.7 \\
28.4\end{array}$ & - & - \\
\hline $\begin{array}{l}30 \\
20 \\
15 \\
12\end{array}$ & $\begin{array}{l}142.0 \\
\end{array}$ & $\begin{array}{l}42 \times 10^{-11} \\
90\end{array}$ & $\begin{array}{l}22 \times 10^{-11} \\
26 \\
80 \\
100\end{array}$ \\
\hline Dark eurrent & 22 & 20 & 16 \\
\hline
\end{tabular}

The effects of adding diaphragms to eliminate stray light were also determined. There are four removable stray light baffles for a given beam size. They are located about 6 in. away from the projection lens and follow two circular iris diaphragms. They can be inserted about every $25 \mathrm{~mm}$ thereafter in the projected beam up to $100 \mathrm{~mm}$ from the center of rotation. They are in all cases slightly larger than the beam dimensions calculated for that location in the box. With the large beam (10 mm wide) no difference could be detected from $13^{\circ}$ just outside of the large beam to $150^{\circ}$ away from the direction of the beam when the stray light baffles were inserted or removed. Later a large cylindrical cell with plane entrance and exit windows and filled with a liquid whose refractive index was not too different from that of the glass was placed on the cell table and again there were no differences when the baffles were in or out of position or over the above angles. However, when the narrow beam $(3 \mathrm{~mm})$ was examined without a cell in place the stray light decreased by about 25 percent below $10^{\circ}$ when appropriate baffles were introduced into the system.

In order to investigate further the effect of stray light the receiver was removed from the phototube housing so that the photomultiplier was receiving light from any angle that its photosensitive surface would allow. Again the addition of the baffles with the large beam while viewing from $45^{\circ}$ to $150^{\circ}$ showed no appreciable change in the response of the phototube. However, visual inspection after long dark adaptation indicated that a baffle in one of the middle positions did decrease some of the diffuse illumination in the photometer. From the results of all these experiments it is felt that this photometer can determine absolute amounts of scattered light from the usual organic liquids without any appreciable error from stray light.

Fluorescence, Polarization, and Monochromatic Filters. The directions of polarization were scribed on the Polaroid filters that were subsequently used for the polarizers. They were then mounted in the instrument by alining the inscribed marks with respect to the perpendicular and horizontal lines at the cell table. The analyzer was then set for extinction of the polarized beam. The percent 
of transmission at extinction for the analyzer was 0.012 and 0.008 for blue light $(436 \mathrm{~m} \mu)$ in the horizontal and vertical direction, respectively, and 0.008 for $546-\mathrm{m} \mu$ light in both the horizontal and the vertical directions. The phototube was found to be equally sensitive to both horizontally and vertically prolonged light of $436 \mathrm{~m} \mu$ wavelength, but was 0.5 percent more sensitive to horizontally prolonged light of $546 \mathrm{~m} \mu$ wavelength.

Four filters for use with fluorescent solutions can be mounted in the barrel of the receiver. The No. 3384 Corning filter transmitted $9 \times 10^{-4}$ percent of the $436-\mathrm{m} \mu$ light, and 88 percent of the $546-\mathrm{m} \mu$ light. The No. 2424 Corning filter transmitted $1.6 \times 10^{-2}$ percent at $546 \mathrm{~m} \mu$ and $7.8 \times 10^{-6}$ at 436 $\mathrm{m} \mu$. These are simply cutoff filters to detect the amount of fluorescence. A set of monochromatic filters similar to those described below could also be used to allow light-scattering measurements to be made on fluorescent solutions.

The monochromatic filter combinations used for the incident beam were examined on a Beckman DK spectrophotometer and found to give less than 0.5 percent of the maximum intensity when measured at $200 \mathrm{~A}$ from 4358 and $5461 \mathrm{~A}$. The transmission of the interference filter was about 2.5 times that of the glass filter.

Neutral Filters. Table 2 summarizes the results of many measurements made over a period of 9 months on the transmission values and their average deviations for the five neutral filters that are fixed in the instrument and moved reproducibly into position. The first four were always checked by the amplifier; whereas the very dense one was usually checked in comparison with another neutral filter and the amplifier. In some experiments a direct measurement of the dense filter transmission was made using the amplifier and gave good agreement with the comparison measurements even though the signal to noise ratio was extremely low. Table 3 gives the transmission results under changing experimental viewing conditions. Also table 2 indicates that the 1P21 and 5819 phototubes give the same transmission values. There is no question but that there is a slight nonuniformity of the transmittance over the total area of the neutral filter, as was seen in later experiments using a small slit to scan the filter; however, all ordinary measurements are carried out as above with fields of view of such dimensions that these small differences are integrated into a constant average value.

TABLE 2. Transmittance of neutral filters for different phototubes at different wavelengths of light

\begin{tabular}{|c|c|c|c|c|}
\hline \multirow{2}{*}{ Filter } & \multicolumn{2}{|c|}{ 1P21 phototube } & \multicolumn{2}{|c|}{5819 phototube } \\
\hline & $436 \mathrm{~m} \mu$ & $546 \mathrm{~m} \mu$ & $546 \mathrm{~m} \mu$ & $436 \mathrm{~m} \mu$ \\
\hline $\begin{array}{l}1 \\
2 \\
3 \\
4 \\
5\end{array}$ & $\begin{array}{c}0.495 \pm 0.6 \% \\
.305 \pm 0.3 \% \\
.0792 \pm 0.5 \% \\
.0190 \pm 0.5 \% \\
6.64 \times 10^{-5} \pm 0.2 \%\end{array}$ & $\begin{array}{c}0.526 \pm 1.5 \% \\
.329 \pm 0.5 \% \\
.0953 \pm 1 \% \\
.0263 \pm 0.4 \% \\
2.22 \times 10^{-4} \pm 0.4 \%\end{array}$ & $\begin{array}{l}0.532 \\
.333 \\
.0971 \\
.0264 \\
2.26 \times 10^{-4}\end{array}$ & $\begin{array}{l}0.502 \\
.305 \\
.0794 \\
.0191 \\
6.80 \times 10^{-5}\end{array}$ \\
\hline
\end{tabular}

TABLE 3. Transmittance of neutral filters at $546 \mathrm{m \mu}$ for various size beams and different fields of view

\begin{tabular}{|c|c|c|c|c|}
\hline \multirow{2}{*}{ Filter } & \multicolumn{3}{|c|}{ 10-mm beam } & \multirow{2}{*}{$\frac{3-\mathrm{mm} \text { beam }}{5.5 \times 9.9 \mathrm{~mm}}$} \\
\hline & $5.5 \times 9.9 \mathrm{~mm}$ & $3.0 \times 7.0 \mathrm{~mm}$ & $5.04 \mathrm{~mm}$ diam & \\
\hline $\begin{array}{l}1 \\
2 \ldots \ldots \\
3 \\
4 \\
5 \ldots\end{array}$ & $\begin{array}{l}0.526 \\
.329 \\
.0964 \\
.0263 \\
2.22 \times 10^{-4}\end{array}$ & $\begin{array}{l}0.527 \\
.329 \\
.0970 \\
.0262 \\
2.19 \times 10^{-4}\end{array}$ & $\begin{array}{l}0.522 \\
.330 \\
.0964 \\
.0264 \\
2.20 \times 10^{-4}\end{array}$ & $\begin{array}{l}0.552 \\
.323 \\
.01016 \\
.0260 \\
2.24 \times 10^{-4}\end{array}$ \\
\hline
\end{tabular}

Table 3 indicates no differences in the transmission values beyond experimental error when only the field of view is changed. However, special attention should be directed to the differences which appear when a beam which is smaller than the field of view of the photometer is used. These differing transmittances are no doubt due to the fact that a neutral filter with nonparallel faces or inexact perpendicular alinement can shift the lightbeam when placed in position. This shift then makes the entire lightbeam strike a different part of the photosensitive surface to give a slightly different signal. This causes no problem as long as the transmittance for such geometries is determined in the apparatus as it is used.

\subsection{Optical}

There are two optical systems to consider in the light-scattering photometer, namely, the projection system and the receiving system. In this instrument both systems are telecentric, that is, the aperture stop is located on the focal plane of the lens so that the principal ray in the projected beam is at all times parallel to the optic axis. ${ }^{5}$ Figure 7 shows the diagram of the system for a lens L, of focal length $f$, an aperture stop $S_{2}$, and a field stop $\mathrm{S}_{1}$. For the projection system the dimensions are: $\mathrm{f}=98.6 \mathrm{~mm}, \mathrm{r}=318 \mathrm{~mm}, \mathrm{x}=48.1 \mathrm{~mm}$. From these dimensions the angular divergence of the beam, represented by one-half of the angle $\alpha$ in the diagram, and the magnification may be computed. It can be shown that with the dimensions in this instrument the angular divergence of the rays from the center

${ }^{5}$ L. Kushner, J. Opt. Soc. Am. 44, 155 (1954).

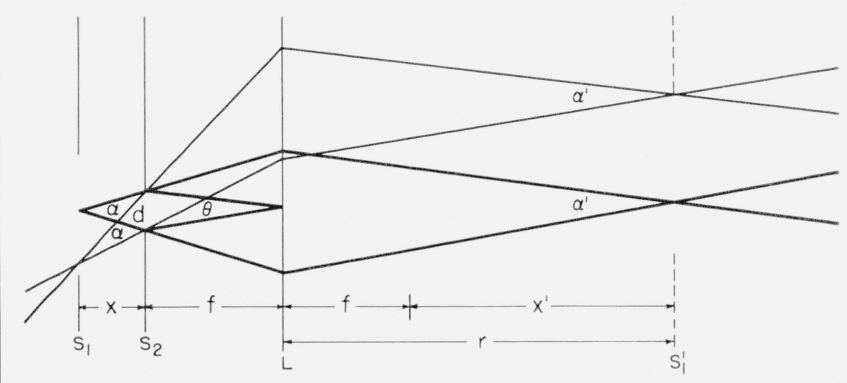

FIGURE 7. A schematic representation of the telecentric system and the angles of bec:m projection or source acceptance. 
to the edge of the field stop differ by no more than 2 percent in the vertical direction with the largest aperture stop ever used, and by much less in the horizontal direction. The angular divergence of the most divergent projected beam is calculated to be $0.28^{\circ}$ in the horizontal and $0.43^{\circ}$ in the vertical direction, although for certain special measurements this beam was replaced by one having a $0.07^{\circ}$ divergence in all directions. Although the divergence is difficult to measure exactly because of the indistinct image away from the field stop $\mathrm{S}_{1}^{\prime}$ the divergence measured $250 \mathrm{~cm}$ away from $\mathrm{S}_{1}^{\prime}$ gave agreement within a few percent of the calculated value.

In order to determine accurately the dimensions of the projected beam, measurements were made in three ways. Visual measurements were made with a telescope focused on a ruled glass scale located on the center of rotation. Then photographs were taken with a camera focused on the scale, and also on the centering fixture used for alining the instrument. The glass scale was particularly useful because it could be immersed in a solvent in a lightscattering cell to make certain of the unchanged magnification when the distance $\mathrm{x}$ in figure 7 was changed slightly to accommodate the decreased optical distance to the center of the cell. The results for three beams measured at the center of rotation are as follows: $9.20 \pm 0.07$ by $11.97 \pm 0.10$ $\mathrm{mm}, 3.09 \pm 0.03$ by $9.22 \times 0.07 \mathrm{~mm}$, and $1.60 \pm 0.03$ $\mathrm{mm}$ diameter. The measured magnification of 2.06 is within 2 percent agreement of the calculated value.

The receiver system may also be represented by figure 7 and has the dimensions $\mathrm{x}=36.50$, and $\mathrm{r}=78$ to $117 \mathrm{~mm}$. The achromatic lens used in the receiver, however, had its principal points determined on a nodal slide in the NBS Optics Division. The value for the equivalent focal length needed to calculate the angular acceptance of the system determined by $\mathrm{S}_{1}$ and $\mathrm{S}_{2}$ was $40.44 \pm 0.10 \mathrm{~mm}$ for $575-\mathrm{m} \mu$ wavelength light. The aperture stop was located on the focal plane within $\pm 0.1 \mathrm{~mm}$. The magnification of the field stop is about 1.11. The entire phototube housing can be moved so that the optical distance $r$ may be kept constant by changing the geometrical distance when large size lightscattering cells are used and compared to quantities measured in air.

The beam uniformity has been tested in many ways. When the lamp is centered on the 1- by 3$\mathrm{mm}$ aperture stop in the projector, the uniformity of the $9.2-$ by $12.0-\mathrm{mm}$ beam as measured with a phototube having a $0.254-\mathrm{mm}$ slit in front arranged to accept about $2.5^{\circ}$ semicone angle, is constant within \pm 0.5 percent over 95 percent of the beam. In this experiment the phototube housing is advanced across the beam by means of a screw with a pitch of 40 threads per inch. Evaluating more than this amount of the beam would require a smaller slit since the edge effects can be calculated to arise somewhere in this region. The same beam size at the center of rotation but having a smaller divergence gives the same results although the lamp is not as difficult to aline since less of the lamp area is required to fill the stop. As might be expected, the smaller size beams give the same uniformity.

Alinement of the receiver and beam direction is necessary for these measurements, and this can only be achieved by the use of mechanical fixtures followed by optical sighting methods. The end-on phototube does not appear as sensitive to extremely small angular deviations from parallelism as the $1 \mathrm{P} 21$.

The authors are indebted to P. Pfaff of the NBS Machine Shop for his many suggestions and excellent workmanship. They also thank E. Webb of the NBS Optical Shop for his construction of many of the optical components, and his cooperation in fitting and mounting many of the other items. 